\title{
Extending Dronacharya's Ball Retrieval method to collect Exam Results announced online After Revaluation
}

\author{
Hariharan Nalatore, Sasikumar. N, Purnimaa. S. Dixit, Prashantha. B. B
}

\begin{abstract}
After the announcement of UG / PG course Exam results by any University, it is routine that some of the students will apply for Revaluation for some of the subjects attempted by them. The number of subjects applied for Revaluation will vary from student to student. So, after Revaluation the University will again announce results online. Here, we will discuss "how a class teacher can collect the revaluation results announced online in an excel file?" and "how to update the same in the old marks excel file?" by considering VTU exam results as an example.
\end{abstract}

Index Terms- Revaluation results, Dronacharya, Asymmetric data, Microsoft Excel, Matlab.

\section{INTRODUCTION}

After the announcement of UG / PG course Exam results by any University, it is routine that some of the students will apply for Revaluation for some of the subjects attempted by them. The number of subjects applied for Revaluation will vary from student to student . For example: the 1st student may apply for revaluation just for one subject, the 2 nd student may apply for 5 subjects, the 3rd student may apply for 2 subjects etc. So, after Revaluation the University will again announce results. In particular, Visvesvaraya Technological University (VTU) [1] announces the Revaluation results online only for the selective subjects applied by the students.

In our earlier paper [2], we had discussed how a class teacher can collect in an Excel file the VTU exams results announced online by Dronacharya's Ball retrieval method. When the number of subjects are same (for example 8 ) for each student, then segregating the result data student wise is easy by developing templates in excel based on mere formulas alone to "Unstuck the Raw data from big Master column" [3]. Since, after Revaluation, the Revaluation results are announced only for the selective-subjects applied by a given student, and as the number of subjects applied for Revaluation varies from student to student hence, cracking a pattern in this asymmetric configuration to align / segregate the marks data student wise is a huge challenge. In this paper,

Hariharan Nalatore, Dept of Physics (VTU R\&D center), Sir M. Visvesvaraya Institute of Technology, Bangalore, India

Sasikumar N, Dept of Physics, Sir M. Visvesvaraya Institute of Technology, Bangalore, India.

Purnimaa S Dixit, Dept of Physics, Sir M. Visvesvaraya Institute of Technology, Bangalore, India.

Prashantha. B. B, Dept of Humanities, Sir MVIT, Bangalore. we will discuss "how to segregate the Revaluation result data student wise from big Master column?" and "how to update the old marks file in the light of Revaluation Results ?’.

\section{II .HOW TO COLLECT THE REVALUATION RESULT RAW DATA} FROM VTU WEB PORTAL?

We can collect the Revaluation Marks data from the VTU web portal and stack them as a big Master column in an Excel file (for example in a file named "Revaluation_Dronacharya_RawData.xlsx") by exactly following the same procedure as described in our previous paper [2]. In this case, the result collected will be in the format (USN, Subject Code, Subject Name, Revaluation Marks, Old Marks, Internal Marks, Result) as shown in the figure 1. The stacked Master column of Revaluation Marks data is as shown in figure 2.

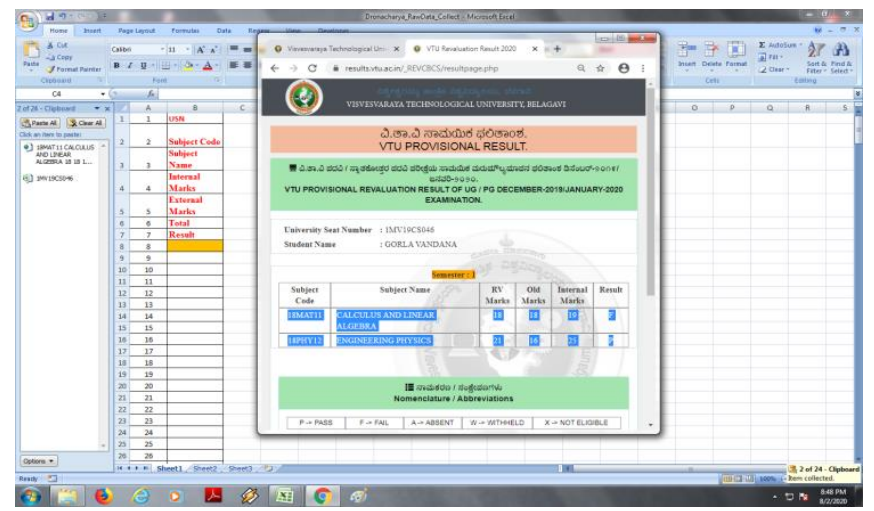

Figure 1: The format of Revaluation result data in VTU web portal

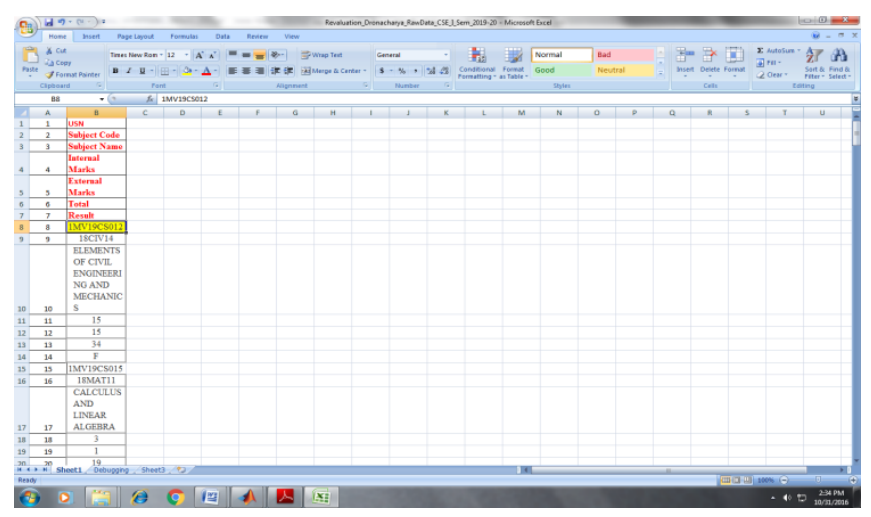

Figure 2: The Revaluation Raw data is a big Master column B 


\section{HOW TO SEGREGATE THE REVALUATION RESULT RAW DATA STUDENT WISE FROM THIS BIG MASTER COLUMN?}

Since here, the number of subjects applied for Revaluation varies from student to student, it is not possible to segregate / align the Revaluation Result Raw data student-wise by using formula based excel templates [3] as described in our previous paper [2]. In order to "Unstack data from big master column"to overcome this limitation, we have developed a computer program using Matlab [4, 5] namely "Format_Asymmetric_Revaluation_ManualEntry_V TU_ExamMarks_Data.m". The entire code is given in Appendix-1.

Here, from the input file (for example: the file named "Revaluation_Dronacharya_RawData.xlsx"), the student wise aligned / segregated data is put in an output excel file (for example: in a file named "'Segregate_RawData_Revaluation_VTU_Exam_data"). An example of student-wise aligned $\bar{d}$ ata is as shown in the figure 3.Further, the student names can be recovered by using official studentlist and applying VLookup formula of Excel as described in our previous paper [2].

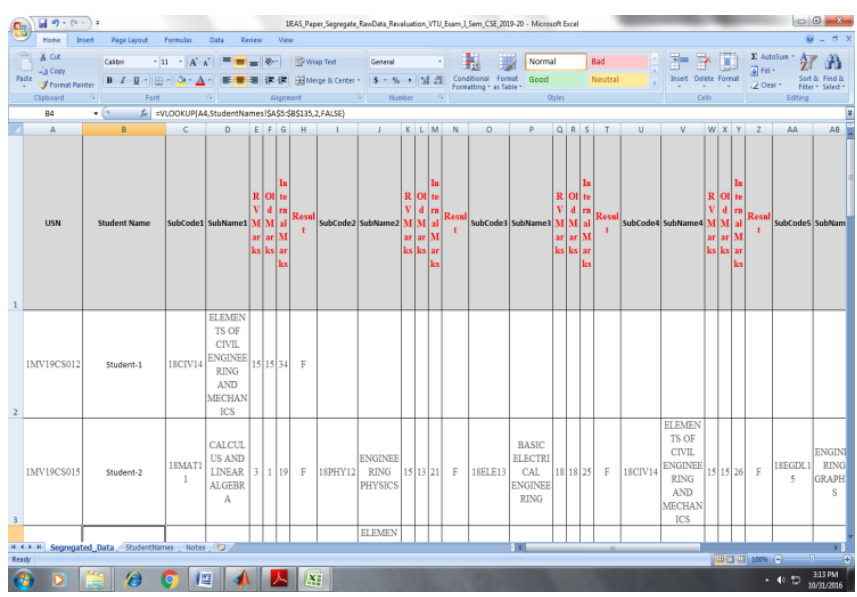

Figure 3: The student wise aligned data.

\section{HOW TO UPDATE THE OLD MARKS FILE USING STUDENT WISE SEGREGATED REVALUATION DATA?}

In order to update the old marks file by using segregated Revaluation data, we have developed a program in Matlab [4, 5] namely "Update_After_Revaluation_Marks_in_Old_Marks_File.m" . This complete Matlab program is given in Appendix-2.

$>$ Here, the input files are old marks file (for example file named

'Segregate_RawData_VTU_Old_Marks_File.xlsx') and segregated Revaluation data (for example a file named

'Segregate_RawData_Revaluation_VTU_Exam_da ta.xlsx').

$>$ The data in the old marks file are in the format (USN, Student Name, SubCode1,SubName1,In,Ex,Tot,Result,SubCode2, SubName2,In,Ex, Tot,Result..... upto 8 subjects).

$>$ The revaluation result data is in the format (USN, Student Name, SubCode1, SubName1,RvMarks,OldMarks,In,Result,
SubCode2,SubName2,RvMarks,OldMarks,In,Resul t... upto 8 subjects).

$>$ The Updated Revaluation Marks are put in an output file named for example'Revaluation_OutPut_LandPad_File_4_VT UResult_Data'

\section{CONCLUSION}

In this paper, we discussed "how to collect and segregate student wise" the Revaluation Results data using Matlab code. We also discussed "how to update / replace" this student wise segregated data in the old marks excel file using Matlab.

\section{APPENDIX}

\section{$\underline{\text { Appendix 1: }}$}

function []

Format_Asymmetric_Revaluation_ManualEntry_VTU_Exa mMarks_Data;

clearall; close all; clc;

\% Read the Manually Generated RawMarks excel file for the first sheet only.

$\%$ Indicate the file names with extensions also.

[NUMERIC_1,TT_dummy1,All_Student_Raw_MarksData] $=$

xlsread('Revaluation_Dronacharya_RawData.xlsx','Sheet1'); clearNUMERIC_1TT_dummy1

$\%$ Note: The no of subjects applied for Revaluation varies from one student to the other.

No_of_Headers = 7;

No_of_Coloumn_for_Each_Subject $=6$;

$\%$ Find total number of students by identifing the row_no's where the USN's

$\%$ are located by using the part of the string that is common in every USN.

$\%$ In this example, the first 5 characters in USN are common for all students.

$\%$ (For example: 1MV19)

\section{$\%$ Initilise the student count}

if

(All_Student_Raw_MarksData\{No_of_Headers+1,2\}(1:5)= $=' 1 \mathrm{MV} 19$ ') $\%$ We start storing data from 8th row onwards. stu_no=1;

USN_location_rowno $\{$ stu_no $\}=$ No_of_Headers +1 ; end

Total_Rows=length(All_Student_Raw_MarksData);

forrow_no $=$ No_of_Headers +2 : Total_Rows

if length(All_Student_Raw_MarksData\{row_no,2\}) $>4$ if

(All_Student_Raw_MarksData $\{$ row_no,2\}(1:5)=='1MV19') stu_no = stu_no +1 ;

USN_location_rowno $\{$ stu no $\}=$ row no; end 
end

end

TotalStudents $=$ stu_no

clearstu_no

$\%$ Allign the students data so that each row gives the marks details of a

$\%$ particular student that he/she had applied for Revaluation.

$\%$ Capture the Marks range for each student with USN also. for $\mathrm{j}=1$ : TotalStudents

$\%$ Get the USN of the jth student and store it in 1st row.

Aligned_All_Student_MarksData $\{\mathrm{j}\}\{1\}=$

All_Student_Raw_MarksData $\{$ USN_location_rowno $\{\mathrm{j}\}, 2\}$;

$\%$ Note: The 2nd index is reserved for student name. This can be

$\%$ found using the Excel's Vlookup function using official student list

$\%$ Capture and store the Marks range

kstart $=$ USN_location_rowno $\{\mathrm{j}\}+1$;

$\%$ Put a check for kend, if $\mathrm{j}==$ TotalStudents

if $\mathrm{j}==$ TotalStudents

kend $=$ Total_Rows $\%$ the last row

else

kend $=$ USN_location_rowno $\{\mathrm{j}+1\}-1$; \% To capture the

Marks range for the last USN also.

end

$\%$ Initilise the counter

countk=2;

for $\mathrm{k}=\mathrm{kstart}:$ kend

countk=countk+1;

Aligned_All_Student_MarksData $\{\mathrm{j}\}\{$ countk $\}=$

All_Student_Raw_MarksData $\{\mathrm{k}, 2\}$;

end

clearcountk

end

clearj

$\%$ Write the Aligned data to an Excel file.

for $\mathrm{j}=1$ : TotalStudents

[SUCCESS,MESSAGE] =

xlswrite('Segregate_RawData_Revaluation_VTU_Exam_dat

a',Aligned_All_Student_MarksData $\{1, \mathrm{j}\}$, 'Sheet1',['A'

num2str(j)]);

End

\section{Appendix 2:}

function []$=$

Update_After_Revaluation_Marks_in_Old_Marks_File;

clearall; close all; clc;

$\%$ Read the Old Marks file (before revaluation)

[NUMERIC_1,TT_dummy1,AllStudent_Old_MarksData]

$=$

xlsread('Segregate_RawData_VTU_Old_Marks_File.xlsx','S egregated_Data');

clearNUMERIC_1TT_dummy1

$\%$ Find total students in that Class

total_students $=$ size $($ AllStudent_Old_MarksData,1) $-1 \%$

one is removed as the 1 st row has header.

total_coloumns $=\operatorname{size}($ AllStudent_Old_MarksData,2)

total_subjects $=8$;
$\%$ Read the After Revaluation Marks from the relevant Excel file

[NUMERIC_2,TT_dummy2,Student_Revaluation_MarksDa ta] $=$

xlsread('Segregate_RawData_Revaluation_VTU_Exam_dat a.xlsx','Segregated_Data');

clearNUMERIC_2TT_dummy2

$\%$ Fine the no of students applied for revaluation. total_Reval_Students =

size(Student_Revaluation_MarksData,1) - $1 \%$ one is removed as the 1st row has header.

$\%$ Update the Revaluation Marks in Old Marks.

$\%$ Check whether a given student has applied for revaluation.

If yes, then

$\%$ identify the subjects applied and update those subject

marks in the old

$\%$ file for that student.

for $\mathrm{i}=1:$ total students

for $\mathrm{j}=1$ : total_Reval_Students

$\%$ The USN will start from 2 nd row as the 1 st row is a header. So,

$\% 1$ is added inside the cell.

ifstrcmp(AllStudent_Old_MarksData $\{\mathrm{i}+1,1\}$,

Student_Revaluation_MarksData $\{\mathrm{j}+1,1\}$ )

for $\mathrm{k}=1$ : total_subjects

for $1=1$ : total_subjects

ifstrcmp(AllStudent_Old_MarksData $\{\mathrm{i}+1,6 * \mathrm{k}-3\}$,

Student_Revaluation_MarksData $\{\mathrm{j}+1,6 * 1-3\}$ )

$\%$ Update the External Exam Marks after revaluation

AllStudent_Old_MarksData $\{\mathrm{i}+1,6 * \mathrm{k}-3+3\}=$

Student_Revaluation_MarksData $\{\mathrm{j}+1,6 * 1-3+2\}$;

$\%$ Compute the new total (Internal + External out of 100) for that subject

AllStudent_Old_MarksData $\{\mathrm{i}+1,6 * \mathrm{k}-3+4\}=$

Student_Revaluation_MarksData $\{\mathrm{j}+1,6 * 1-3+2\}+$

Student_Revaluation_MarksData $\{\mathrm{j}+1,6 * 1-3+4\}$;

$\%$ Get the updated result from Reval data.

AllStudent_Old_MarksData $\{\mathrm{i}+1,6 * \mathrm{k}-3+5\}=$

Student_Revaluation_MarksData $\{\mathrm{j}+1,6 * 1-3+5\}$;

end

end

end

end

end

end

Updated_AllStudent_Reval_MarksData =

AllStudent_Old_MarksData;

clearijk1

$\%$ Write this updated Revaluation Marks to an output file [SUCCESS,MESSAGE] =

xlswrite('Revaluation_OutPut_LandPad_File_4_VTUResult Data',Updated_AllStudent_Reval_MarksData);

\section{REFERENCES}

[1] HTTPS://VTU.AC.IN/

[2] “Collecting The EXAm Results AnNounced OnLine By DRONACHARYA'S BALL RETRIEVAL METHOD”, INTERNATIONAL 
JOURNAL OF ENGINEERING AND APPLIED SCIENCES (IJEAS), VOL-7, ISSUE-3, MARCH 2020

[3] "UNSTACKING DATA IN EXCEL",

HTTPS://WWW.YOUTUBE.COM/WATCH?V=RSCXNULRSH0

[4] “GetTing STARTED With MATLAB: A QUick INTRODUCTION For SCIENTISTS \&ENGINEERS", RUDRAPRATAP, OXFORD UNIVERSITY PRESS, 2010.

[5] Https://Www.Mathworks.Com/

\section{Authors profile}

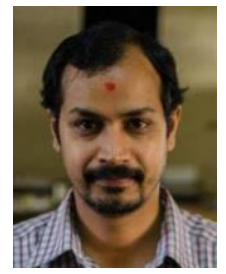

Dr. Hariharan Nalatoreobtained his Ph.D from Indian Institute of Science, Bangalore in 2005.He is currently working as an Associate Professor in the department of Physics, Sir MVIT, Bangalore. His research interest includes Statistical Signal processing, Data Analysis, Computer Vision among others. He has published 6 research papers in International Journals.

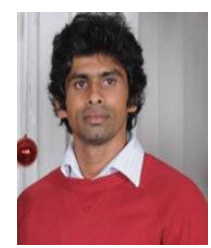

Sasikumar Nobtained his M.Sc in Physics from Bangalore University in the year 2000. He is working as an Assistant Professor in the department of Physics, Sir MVIT. His research interest includes Data Analysis of Neurobiological signals, Computer Vision. He has published 2 papers in International Journals.

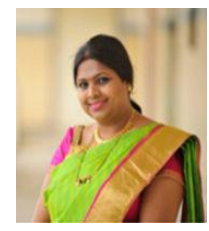

Purnimaa S Dixitobtained her M.Sc in Physics from Bangalore University in the year 2000. She is working as an Assistant Professor in the department of Physics, Sir MVIT. Her research interest includes Office-Automation, Nano-materials and Data Analysis. She has few publications to her credit in reputed journals.

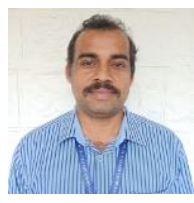

Prashantha. B. B obtained his M.A in Political Science. He is working as an Assistant Professor in the department of Humanities. His areas of interest includes Legal and Business Environment including "Business Law, Industrial Relations and Legislations", Kannada Literature and Office Automation. 